Louisiana State University

LSU Digital Commons

$1-1-1996$

\title{
A passive vibration isolation stack for LIGO: Design, modeling, and testing
}

Joseph Giaime

LIGO, Massachusetts Institute of Technology

Partha Saha

LIGO, Massachusetts Institute of Technology

David Shoemaker

LIGO, Massachusetts Institute of Technology

Lisa Sievers

California Institute of Technology

Follow this and additional works at: https://digitalcommons.Isu.edu/physics_astronomy_pubs

\section{Recommended Citation}

Giaime, J., Saha, P., Shoemaker, D., \& Sievers, L. (1996). A passive vibration isolation stack for LIGO:

Design, modeling, and testing. Review of Scientific Instruments, 67 (1), 208-214. https://doi.org/10.1063/ 1.1146573

This Article is brought to you for free and open access by the Department of Physics \& Astronomy at LSU Digital Commons. It has been accepted for inclusion in Faculty Publications by an authorized administrator of LSU Digital Commons. For more information, please contact ir@lsu.edu. 


\title{
A passive vibration isolation stack for LIGO: Design, modeling, and testing
}

\author{
Joseph Giaime, ${ }^{\text {a) }}$ Partha Saha, and David Shoemaker \\ LIGO Project, Massachusetts Institute of Technology, Bldg. 20B-145, Cambridge, Massachusetts 02139 \\ Lisa Sievers \\ LIGO Project, California Institute of Technology, Pasadena, California 91109
}

(Received 5 September 1995; accepted for publication 10 October 1995)

\begin{abstract}
Multiple-stage seismic vibration isolation stacks, which consist of alternating layers of stiff masses and compliant springs, can provide significant passive filtering of ground vibration for experiments and equipment that are sensitive to mechanical noise. We describe the design, modeling and testing of a prototype of a stack suitable for use in the Laser Interferometer Gravitational-wave Observatory (LIGO). This is a four-stage elastomer (spring) and stainless steel (mass) stack, consisting of a table resting on three separate legs of three layers each. The viscoelastic properties of elastomer springs are exploited to damp the stack's normal modes while providing rapid roll-off of stack transmission above these modal frequencies. The stack's transmission of base motion to top motion was measured in vacuum and compared with three-dimensional finite-element models. In one tested configuration, at $100 \mathrm{~Hz}$, horizontal transmission was $10^{-7}$, vertical transmission was $3 \times 10^{-6}$, and the cross-coupling terms were between these values. (C) 1996 American Institute of Physics. [S0034-6748(96)03801-X]
\end{abstract}

\section{INTRODUCTION}

Long baseline interferometric gravitational wave detectors are being planned by several groups throughout the world $^{1,2}$ with the expectation that a network of detectors will be in operation by the end of this decade. The detectors are based on the interferometric measurement of gravitational wave (GW) strains induced between freely suspended masses. ${ }^{3}$ The anticipated magnitude of the gravitational strains to be detected by these instruments from astrophysical sources is smaller than $10^{-21}$, or equivalently, $4 \times 10^{-18}$ $\mathrm{m}$ of relative motion of the suspended masses over a $4 \mathrm{~km}$ baseline, approximately $10^{-8}$ of the diameter of an atom. The techniques to measure such small motions interferometrically have been developed over the past two decades in short baseline prototype laser interferometers ${ }^{1,2,4}$ for motions with frequencies above approximately a few hundred hertz.

Current thinking in astrophysics suggests that gravitational wave sources will most likely emit most strongly at frequencies of several hundred hertz and lower. The best understood sources, coalescing binary neutron stars or black holes, will spend a few seconds emitting a gravitational wave chirp with frequencies from tens to hundreds of hertz before coalescence. The formation of hundred-solar-mass black holes would produce gravitational radiation in a broad band around a hundred hertz. The primordial gravitational wave background resulting from the initial moments in the expansion of the universe may be observable in the ten to several hundred hertz band.

At these frequencies the sensitivity of the long baseline detectors is limited more by random forces imparted to the suspended masses than by the ability to sense small motions. The dominant sources of random motion are thermal excita-

a) Present address: University of Colorado, JILA Campus Box 440, Boulder, CO 80309. tions in the suspension that supports the masses, and random accelerations induced by seismic vibrations at the suspension supports. For frequencies below a few hertz, the naturally occurring and unshieldable Newtonian gravitational gradient forces on the suspended masses, due to mass motion in the ground and atmosphere, impose a limit to the sensitivity of terrestrial long baseline detectors. ${ }^{6}$

Seismic accelerations at the suspension supports do not constitute a fundamental limit to the sensitivity of gravitational wave detection. The reduction of seismic motion, since it is an acceleration relative to the inertial frame, is amenable to creative engineering. The overall strategy to reduce the influence of seismic accelerations in gravitational wave interferometers involves several elements. Each test mass is suspended with fine wires; these pendulums have low mechanical dissipation to minimize thermal noise. The suspension affords significant isolation from seismic accelerations as well as the thermal noise driving the suspension supports, ${ }^{5,7,8}$ but the largest reduction in seismic accelerations is provided by additional vibration isolation systems that establish a seismically quiet platform for the suspensions. Vibration isolation systems are broadly classified as passive and active. Passive systems are assemblies of springs and masses arranged to attenuate the transmission of seismic accelerations. The transmission is frequency dependent with attenuation above the resonant frequency of the mass spring combinations. ${ }^{7,9,10}$ Active systems measure the seismic motions and then provide forces applied to the suspension supports to cancel the seismic accelerations. ${ }^{11,12}$

This article describes the concept, modeling, and tests of a passive vibration isolation stack. It has been developed to improve the low-frequency performance of current interferometric prototype detectors and, with further modifications, may become one element of the vibration isolation system for the initial laser interferometer gravitational-wave observatory (LIGO) detector. The major virtue of the system is its simplicity and reliability. 


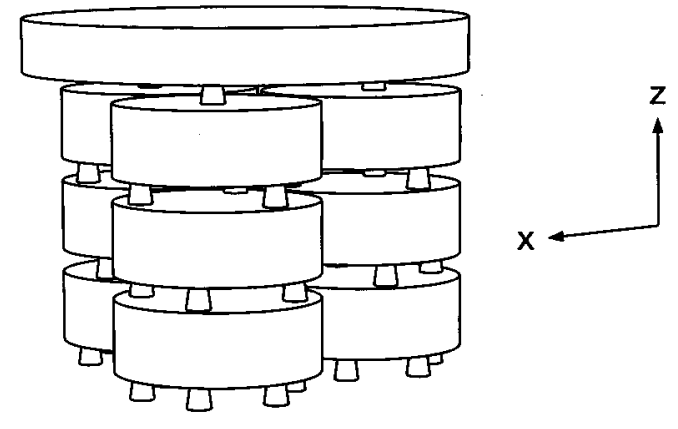

FIG. 1. Perspective drawing of the prototype isolation stack. A single circular table is supported through springs by three isolating legs of three mass-spring layers each. The base table is not shown. The overall height is $68 \mathrm{~cm}$; the width, $78 \mathrm{~cm}$.

The stack design described here is based upon the following three features: elastomer springs, compact mass elements, and separated legs. Vacuum-compatible elastomers are used as the spring material. Elastomers have internal viscoelastic damping, which reduces the mechanical quality factors $(Q)$ of both the resonances within the spring elements and the overall resonances of the stack structure, without significantly compromising high-frequency isolation. Second, the masses are sufficiently compact and stiff so that their internal resonances have frequencies above the important GW band. Finally, the stack consists of separate legs of mass-spring layers, upon which a single table rests. This design allows the compact masses to be incorporated in a larger overall structure that avoids structural elements with parasitic vibrational modes within the GW band.

\section{THE DESIGN}

\section{A. Isolation stack design approach}

The basic stack design may be thought of as four cascaded harmonic oscillators. The lower three oscillators consist of layers of three stainless steel cylinders resting on layers of elastomer springs, forming three cylindrical legs. The final layer is a single circular aluminum table supported through three elastomer springs on the three legs. See Fig. 1. The properties of the configurations tested are listed in Table I.

The stack's vertical $(z)$ response on the top table to vertical excitation at the base, defined as $T_{z z}(f)$, can be accurately described as four cascaded one-dimensional oscillators. At frequencies well below a single oscillator's resonant frequency, $f_{0}$, there is unity transmission. Near resonance, the transmission is enhanced by approximately the $Q$ of the oscillator. Well above the resonance, the transmission falls with frequency as $\left(f_{0} / f\right)^{n}$, where $n$ lies between 1 and 2 and depends on the dissipation mechanism in the springs and on the $Q$. When four such layers are cascaded, the resonance peaks in $T_{z z}(f)$ will be spread in frequency and will correspond with the frequencies of the four rigid-body modes that have predominantly vertical mode shapes. Well above the resonances, $T_{z z}(f)$ will fall approximately as the product of the individual stages' responses.
TABLE I. Properties of experimentally tested stack configurations. Al $=$ aluminum table, $\mathrm{SS}=3$ stainless steel cylinders, $\mathrm{Fl}=3 \mathrm{M}$ fluorel 2176 , and $\mathrm{RTV}=\mathrm{GE}$ RTV615 silicone rubber.

\begin{tabular}{|c|c|c|c|c|c|}
\hline Layer & Base & 1 & 2 & 3 & 4 \\
\hline Mass element & $\mathrm{Al}$ & SS & SS & SS & $\mathrm{Al}$ \\
\hline \multicolumn{6}{|l|}{ Spring material: } \\
\hline stack A & & $\mathrm{Fl}$ & $\mathrm{Fl}$ & $\mathrm{Fl}$ & $\mathrm{Fl}$ \\
\hline stack B & & $\mathrm{Fl}$ & $\mathrm{Fl}$ & RTV & RTV \\
\hline \multirow[t]{2}{*}{ Number of springs } & & 18 & 12 & 9 & 3 \\
\hline & & \multicolumn{2}{|c|}{ Stack A } & \multicolumn{2}{|c|}{ Stack B } \\
\hline \multirow{2}{*}{$\begin{array}{c}\text { Transmission } \\
\text { at } 100 \mathrm{~Hz}\end{array}$} & $T_{x x}$ & \multicolumn{2}{|c|}{$3.2 \times 10^{-6}$} & \multicolumn{2}{|c|}{$1.0 \times 10^{-7}$} \\
\hline & $T_{z z}$ & \multicolumn{2}{|c|}{$1.0 \times 10^{-4}$} & \multicolumn{2}{|c|}{$2.9 \times 10^{-6}$} \\
\hline Peak & $T_{x x}$ & \multicolumn{2}{|c|}{5.3 at $2.0 \mathrm{~Hz}$} & \multicolumn{2}{|c|}{9.4 at $1.8 \mathrm{~Hz}$} \\
\hline Transmission & $T_{z z}$ & \multicolumn{2}{|c|}{4.2 at $8.5 \mathrm{~Hz}$} & \multicolumn{2}{|c|}{11.3 at $6.9 \mathrm{~Hz}$} \\
\hline
\end{tabular}

The stack's horizontal $(x)$ response to horizontal excitation, $T_{x x}(f)$, also shows damped resonant peaks followed by rapid roll-off with frequency. The exact form is not as easy to predict with simple arguments as the vertical response, since $x$ drive couples into modes involving both tilts and translation of the solid elements; the numerical analysis, which is required to study the horizontal motions, is discussed below. There are also high-frequency internal modes in the solid mass and spring elements. The approach we have taken in this design was to make the overall structure (rigid-body) modes low $Q$, to place them below the frequencies where we need high isolation, and to make all of the internal modes high enough in frequency to ensure that they would be well filtered by the rest of the stack, despite their high $Q$. This led to compact structures of steel to be used as the masses, and elastomers as the springs.

\section{B. Vibration transmission specifications}

\section{Stack transfer functions}

To describe the isolation characteristics we first set the notation. If the stack's base and top structures are rigid at the frequencies being considered, motion may be described by three displacement and three angle coordinates. Let $z$ describe vertical, and $x$ and $y$ horizontal displacements. We label the angles as rotations about each of the displacement coordinate axes, $\theta_{x}, \theta_{y}$, and $\theta_{z}$. Displacements at the base and top will be written as vectors in terms of these coordinates, called $X$. The vibration transmission from bottom to top, $T_{i j}(f)$, is therefore a $6 \times 6$ matrix of complex functions of frequency, with $i$ and $j$ ranging over the displacement and angle coordinates. Transmission may be described in the frequency domain because the force-displacement relations for the system's components are linear over the approximately $10^{-6} \mathrm{~m}$ motions encountered in our tests. Motions are transmitted from the base (drive) of the stack to the top as

$$
X_{i, \text { top }}(f)=T_{i j}(f) X_{j, \text { drive }}(f) .
$$

For simplicity, and taking into account the roughly cylindrical symmetry of the stack, transmission transfer functions will hereafter be written as if motion could be completely resolved in terms of the vertical coordinate, $z$, and an arbitrary horizontal coordinate, $x$ : 


$$
\left(\begin{array}{c}
x_{\text {top }}(f) \\
z_{\text {top }}(f)
\end{array}\right)=\left(\begin{array}{c}
T_{x x}(f) T_{x z}(f) \\
T_{z x}(f) T_{z z}(f)
\end{array}\right)\left(\begin{array}{c}
x_{\text {drive }}(f) \\
z_{\text {drive }}(f)
\end{array}\right),
$$

where the drive and top displacement vectors represent the displacement applied to the bottom of the stack and measured at the top of the stack, respectively. However, as is described below, our drive platform can couple horizontal motion to tilt. We will use the tilt angle $\theta_{y}$, hereafter referred to as $\theta$, to supplement the description in terms of $x$ and $z$, and we will model $T_{x \theta}$ and $T_{\theta \theta}$.

\section{Attenuation in the gravity wave band}

We assume the seismic noise that drives the stack to be isotropic with the following spectral density, based on typical measurements at quiet sites: $10^{-9} / f^{3} \mathrm{~m} / \sqrt{\mathrm{Hz}}$ from 0.1 to 1 $\mathrm{Hz}, 10^{-9} \mathrm{~m} / \sqrt{\mathrm{Hz}}$ from 1 to $10 \mathrm{~Hz}$, and $10^{-7} / f^{2} \mathrm{~m} / \sqrt{\mathrm{Hz}}$ from $10 \mathrm{~Hz}$ to $10 \mathrm{kHz} .^{1,13}$

The GW detector is principally sensitive to motion along an $x$ (horizontal) axis, thus constraining $T_{x x}$ and $T_{x z}$. The earth's curvature over the $4 \mathrm{~km}$ length of the LIGO gives a coupling in the pendulum suspension from motion in the $z$ axis to motion along the $x$ sensing axis of at least $3 \times 10^{-4}$, constraining $T_{z z}$ and $T_{z x}$. This is because the direction of the earth's gravity is slightly different at each end point of the interferometer.

We require that the seismic contribution to LIGO's expected noise spectrum not significantly influence the detection rate of predicted GW sources. The seismic noise contribution falls very rapidly with frequency in the region above the stack resonances. A competing noise below about $200 \mathrm{~Hz}$ is expected from the thermal excitation of the mirror pendulum suspension. ${ }^{1,14,15}$ Above $200 \mathrm{~Hz}$, "shot noise," or photon counting statistics, will dominate. An important figure-ofmerit for our seismic isolation system is the crossover frequency at which its noise contribution equals that of the expected thermal noise of the pendulum and test-mass design. This is a general procedure for setting the isolation goal; the requirement for LIGO to which this reasoning leads will evolve as our estimates of other noise sources improve.

With this in mind, a goal for this prototype stack is that the seismic-thermal noise crossover be below $100 \mathrm{~Hz}$. This results in a transmission of $10^{-5}$ or less at frequencies above $100 \mathrm{~Hz}$ for $T_{x x}$ and $T_{x z}$, and approximately a factor of 10 greater transmission for $T_{z x}$ and $T_{z z}$.

\section{Motion amplification at rigid-body resonances}

Rigid-body resonances are those for which the solid masses of the stack behave as nondeforming bodies applying forces to one another through the low-mass spring elements. The transmission peaks due to rigid-body stack resonances, in the region below $20 \mathrm{~Hz}$, where the ground noise is very large, will amplify the motions at those frequencies. The gain and dynamic range of the control systems which maintain the GW detector optical sensor at the operating length and alignment must be sufficient to suppress these motions satisfactorily. Since the low-frequency (below $1 \mathrm{~Hz}$ ) motion dominates the integral of the noise spectral density $\left(x_{\text {rms }}\right)$, high- $Q$ peaks at several hertz do not contribute significantly to $x_{\text {rms }}$, if one only considers "stationary" random ground noise. However, impulse or step excitations might excite high- $Q$ resonances to an unacceptable level. The optical and control systems may contain nonlinearities, which can lead to up-conversion of motion at low frequencies to signals at GW frequencies. Also, noise may be coupled to the mirrors through magnetic fields, which push the small mirror control magnets; any eddy currents induced in the isolation stack would contribute in proportion to the stack structure's velocity, increasing the contribution from the rigid-body modes. These considerations suggest a design with transmission peaks of having $Q$ 's less than about ten.

\section{Selection of spring materials}

Since interferometric gravity wave detectors will operate at pressures less than $10^{-6}$ Torr, the spring elastomers must have low outgassing properties, and the contamination of nearby low-loss optics must be held to an acceptable level. ${ }^{16}$ Metal springs have been proposed ${ }^{10,17}$ which then require the addition of a damping force (e.g., magnetic eddy-current damping), or the use of encapsulated lossy materials which could otherwise have been incompatible with our vacuum requirements.

Fluorocarbon elastomers, often known by their trademark names Viton and Fluorel, are widely used as seal materials for high vacuum systems. We investigated the dynamic properties of 3M-Fluorel 2176 by measuring the real and imaginary parts of the elastic modulus over a range of relevant frequencies. For the frequency region in which we expect rigid-body resonances, $1-25 \mathrm{~Hz}$, the ratio of real to imaginary modulus is approximately 3 . This means that a harmonic oscillator using this spring material will have resonance $Q$ 's of that order as well. The imaginary part of the modulus grows approximately as the square root of excitation frequency, while the real part of the elastic modulus grows more slowly with frequency. By contrast, a system consisting of a viscous (velocity-proportional) damper in parallel with a Hooke-law spring, if analyzed in terms of a complex spring constant, would have an imaginary part linearly proportional to the drive frequency. An advantage of viscoelastic damping for our application is that while the rigid-body resonance peaks are well damped, sufficiently above these modal frequencies, the transmission falls nearly as $\left(f_{0} / f\right)^{2}$ per oscillator stage, where $f_{0}$ is the oscillator's resonance frequency. The transmission of a viscously damped system with a similar resonance $Q$ would fall less steeply, providing less high frequency isolation.

The elastic modulus of GE RTV615 silicone rubber (RTV) is lower than that of the Fluorel, and primarily real, indicating that a mechanical oscillator made with RTV springs would have a $Q$ significantly higher than one with Fluorel. The use of a combination of these materials allows a trade-off to be made between the high damping and low rigid-body-mode resonant frequencies. The vacuum properties of both Fluorel and RTV are currently being studied for this application. ${ }^{16}$

Although the modulus vs frequency data allow prediction of the system's dynamic response, lossy elastomers such as Fluorel flow in response to constant stress. For times of hundreds of seconds and longer, Fluorel exhibits logarithmic 
creep; there is also relaxation over tens of seconds not well described by either the modulus vs frequency data or the long-term drift phenomenon. These nonlinear effects can be maintained at an acceptable level by limiting the stress; we did not observe these effects in the RTV.

\section{EXPERIMENTAL RESULTS}

\section{A. Configurations Tested}

The stack (Fig. 1) leg elements were made from $12.7 \mathrm{~cm}$ long by $35.6 \mathrm{~cm}$ diameter cylinders of 304 stainless steel. These pieces were approximately $100 \mathrm{~kg}$ each, and we calculate that all of the internal resonances lie above $3 \mathrm{kHz}$. Elastomer springs are held in place between the masses by friction. The Fluorel springs are approximately $5.2 \mathrm{~cm}$ tall, with lower and upper diameters of 4.1 and $3.1 \mathrm{~cm}$; for the RTV springs, these numbers are $3.2,3.9$, and $3.2 \mathrm{~cm}$. The top table, made of aluminum, measures $78.5 \mathrm{~cm}$ in diameter and $9.5 \mathrm{~cm}$ in height, with a webbed interior, and weighs $78 \mathrm{~kg}$. A second similar table was used as the platform on which the entire stack was constructed. Table resonances were measured at 467 and $825 \mathrm{~Hz}$, with $Q$ 's of approximately 500 .

Results from tests of two spring configurations are presented. "Stack A" was assembled with springs made with 70 durometer Fluorel. ${ }^{18}$ The "stack B" configuration was identical to the first in the lower two layers, but used RTV springs for the upper layers. See Table I.

\section{B. Dynamic measurements}

The stack's dynamic response was measured over the frequency range $1-350 \mathrm{~Hz}$, in vacuum to prevent acoustic coupling. Three types of measurements were performed.

\section{Mid-frequency measurements}

These measurements were made in the frequency range of approximately 20 to $90 \mathrm{~Hz}$, covering the part of the transfer function over which the vibration transmission drops from about a few tenths to about $10^{-4}$. The stack was built on a base table which itself was resting on three stiff springs. Accelerometers were attached to reference points on the lower table and top table. A frequency-swept sinusoidal force was applied to the bottom table using electromagnetic shakers. Vertical drive was used to measure the complex transfer functions $T_{z z}$ and $T_{x z}$, and horizontal drive was used to measure $T_{x x}$ and $T_{z x}$. The shakers produced motions of about $10^{-6} \mathrm{~m}$ on the drive table, and about $10^{-12} \mathrm{~m}$ on the top table at $100 \mathrm{~Hz}$. We note that the $100 \mathrm{~Hz}$ drive from ground noise is expected to be only of the order of $10^{-11} \mathrm{~m} / \sqrt{\mathrm{Hz}}$, and so the elastomers will not be tested with motions as small as those in operation until the stack is installed in an interferometer.

\section{Low-frequency ground-noise-driven measurements}

At low frequencies the stack's response is strongly influenced by the exact shapes and frequencies of the rigid-body modes, which range in frequency from approximately 1.5 to $25 \mathrm{~Hz}$. The configuration modeled on the computer had the base fixed to a very large reaction mass. When the stack's base table is placed on springs, the low-frequency modes change frequency and shape. To obtain the true stack response below about $20 \mathrm{~Hz}$, the stack was rigidly attached to grounded mounting posts, and driven directly by ground noise. Only two transfer functions were taken by this method: $T_{z z}$ and $T_{x x}$. The close correlation between $x$ and $z$ ground noise did not allow measurement of all four components, so the cross coupling terms $\left(T_{x z}\right.$ and $\left.T_{z x}\right)$ do not appear below $20 \mathrm{~Hz}$ in the transfer function plot of stack A, Fig. 2(a). Stack B has somewhat lower normal mode frequencies, and begins to isolate well at lower modal frequencies, as can be seen in Fig. 2(b), so the cutoff for these low-frequency measurements was lower.

\section{High-frequency cantilever measurements}

In the highest frequency range, above $90 \mathrm{~Hz}$ for stack A, and $60 \mathrm{~Hz}$ for stack $\mathrm{B}$, the stack's attenuation makes the signal on the top table comparable to accelerometer magnetic pickup and amplifier noise. This occurs at a transmission of about $10^{-4}$. To increase the signal size, the accelerometer is mounted on the end of an aluminum cantilever, providing mechanical amplification. The length (and thus the resonant frequency) is stepped through the measurement frequencies while under vacuum via remote-control motors. The $Q$ as driven through the stack under vacuum is taken to be the mechanical amplification factor at the resonance peak. The accuracy of this procedure was verified by comparison with direct measurements at the lower frequencies.

\section{Summary of measured transfer functions}

The largest transmission component in each spring configuration is $T_{z z}$. At frequencies higher than two discernible resonance peaks, $T_{z z}$ falls slightly less steeply with frequency than $f^{-8} \cdot T_{x x}$ also has at least two low-frequency resonances before dropping at higher frequencies at about the same rate as $T_{z z}$, displaced downward in magnitude by a factor of about 20 . The frequencies at which the power-law roll-off begins for stack B are typically about $2 / 3$ of those for stack A.

Substituting RTV for the Fluorel in the top layers significantly enhances the isolation, while only increasing the resonance amplification by a factor of about 2 . We attribute the lack of peak enhancement to the involvement of the remaining high-loss Fluorel springs in all normal-mode motion. The alternative of a more compliant Fluorel spring design is less attractive because of the resulting greater flow (inelastic deformation) upon loading which would result.

\section{Drift measurements}

The stacks exhibit creep under load. To maintain the creep at an acceptable level, we kept the strain in the Fluorel below about $20 \%$. To characterize the creep and the temperature dependence of the overall stack height, position sensors were used to measure the distance between the top and bottom plate of the assembled stack at three points around the circumference. Since prior tests of the individual elastomers had indicated Fluorel would dominate the drift, the complete multilayer stack A was measured. Data were taken for an approximately 10-day period, about 90 days after the initial 


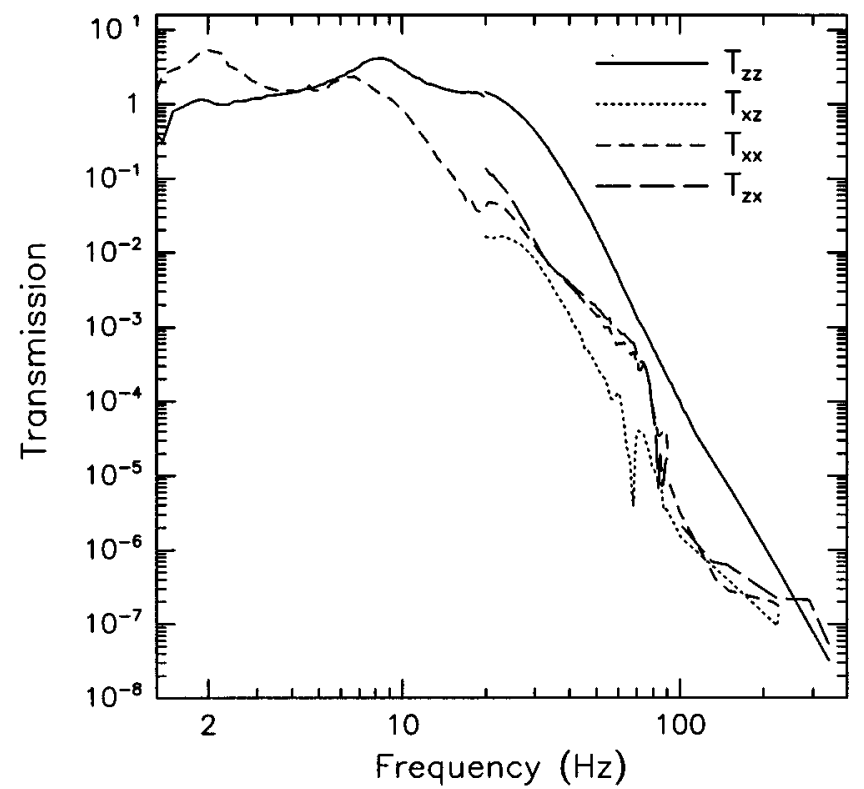

(a)

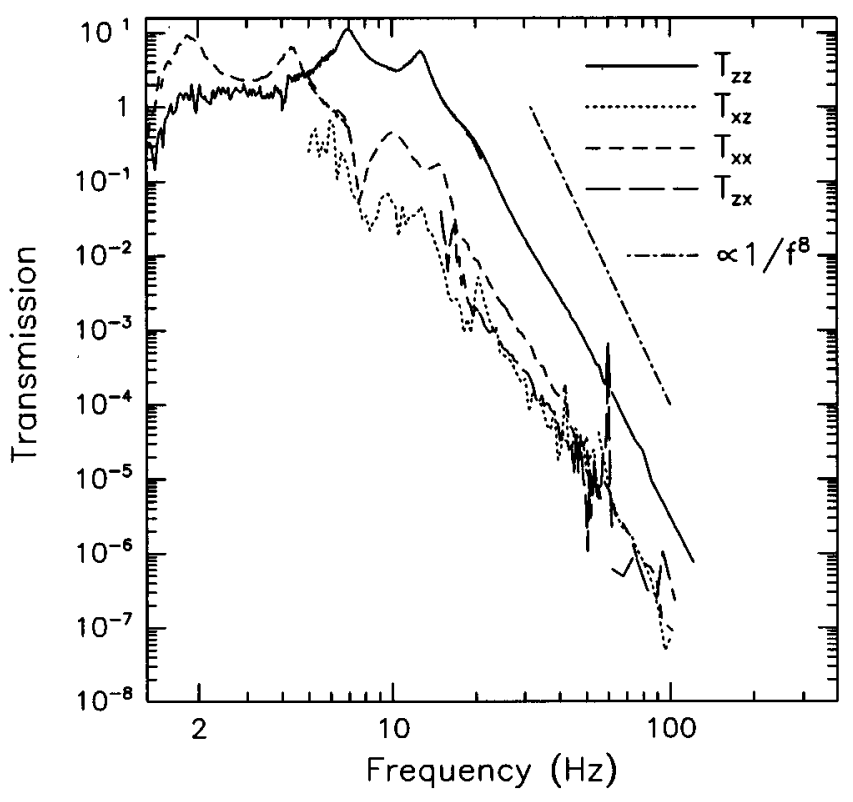

(b)

FIG. 2. Measured transmission matrix elements for (a) stack A, built with all Fluorel springs, and (b) stack B, built with two lower layers of Fluorel springs and two upper layers of RTV springs. Note that in (b), all transfer function components lie below $3 \times 10^{-6}$ at $100 \mathrm{~Hz}$. A line showing the slope of a $1 / f^{8}$ dependence is drawn for comparison with the stack B data.

construction of the stack. The data exhibit a drift of $1.3 \times 10^{-10} \mathrm{~m} / \mathrm{s}$ and a linear response to temperature of $3.2 \times 10^{-5} \mathrm{~m} /{ }^{\circ} \mathrm{C}$ (the stack becomes shorter with increasing temperature). See Fig. 3.

In separate long-term tests of Fluorel under load, we have seen logarithmic drift, which can be expressed as a change in height $\delta h$ of the stack evolving with time $t$ as $\delta h \propto \log (t)$. Under the assumption that elastomer springs be-

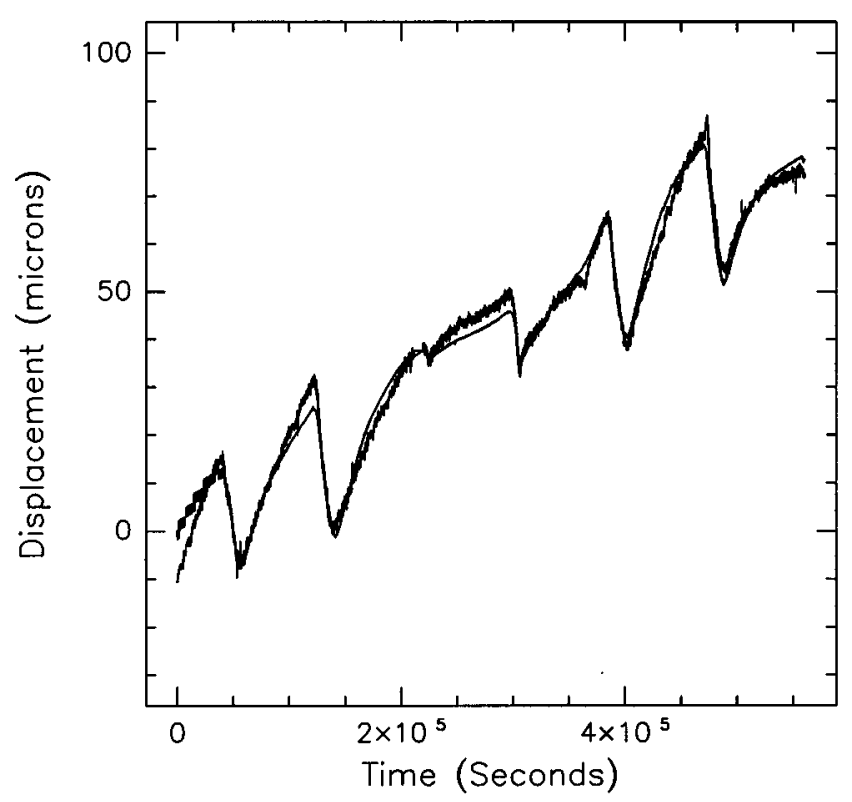

FIG. 3. Drift in the overall height of the stack, data (jagged line) and fit. Daily variations in temperature $\left(\approx 1{ }^{\circ} \mathrm{C}\right.$ peak-to-peak $)$ lead to $\approx 25 \mu \mathrm{m}$ height changes. In addition, over the roughly 7-day period shown, a monotonic shortening of the stack is evident.

have logarithmically in the stack, the drift after an additional year would be $<2 \mathrm{~mm}$ for the complete multilayer stack; if the drift rate were constant (the likely worst case) there would be an $\approx 4.1 \mathrm{~mm} / \mathrm{yr}$ height change. This drift rate can be easily compensated with periodic adjustments to the height of the isolation system support structure.

\section{FINITE-ELEMENT MODELING AND COMPARISON WITH DATA}

\section{A. Pre-experiment model}

To test static stability and to determine the system's response to dynamic excitation, finite-element modeling was carried out using the software package ABAQUS. ${ }^{19}$ This program allows the elastomer behavior to be calculated with a frequency-dependent complex elastic modulus taken from a lookup table assembled from measured material data.

A preliminary model was constructed to test the feasibility of the basic design concept. In particular, we wanted to probe the effects of the various rigid-body and internal structural resonances on the stack transmission, as well as the stability against buckling under load. The intermediate masses in the legs and the top table were composed of arrays of quadratically interpolated solid isoparametric elements taken from the ABAQUS element library. A sufficient number of elements were used to allow the internal resonances of these parts to influence the stack dynamic response.

The results of this preliminary model were the following. The viscoelastic springs adequately damped the rigidbody modes of the stack. The top table's resonances, which in this model began at a few hundred hertz, resulted in high- $Q$ spikes in the transfer function, only slightly damped by the table's contact with the elastomer. The internal reso- 
nances of the leg blocks were above the frequencies of interest, so were not studied in detail, but were seen to produce a leveling of the frequency response as they became important. The model was also tested for buckling stability using nonvertical loading; no instability was seen. From these results we were able to settle on a final stack design with small and stiff leg elements and top table, so that any resonances would occur where there is significant isolation, and with viscoelastic springs to damp the rigid-body modes.

\section{B. Post-experiment model}

A series of models was constructed to reflect the geometry, spring placement and properties of the stacks as built. First, an idealized model was constructed, in which identical springs and identical intermediate masses are placed symmetrically below the top table, and in which the bottoms of the lower springs are mathematically constrained to move together with a driven point-mass base. These symmetries were relaxed in further simulations of stack B. This was done to study the effects of physical imperfections in the stack and testing apparatus, to place limits on the sizes of imperfections that can be tolerated in the design, and to better understand the limitations of the finite-element modeling process.

\section{Spring variations}

In the symmetric model the uniform spring stiffness and placement fails to reproduce several features of the measured stack transmission. For example, the coupling of vertical drive to horizontal motion on top, $T_{x z}$, is nearly zero in the model.

We simulated two kinds of spring variation of the Fluorel springs in stack B: nonuniform spring constants and orientation. Random variation of stiffness among the springs was modeled by introducing normally distributed elastic modulus magnitudes, with a magnitude of standard deviation $5 \%$ roughly based on observed spring-to-spring variation. Crooked springs were implemented as additional springs with cross coupling terms. These perturbation springs caused a force in the $z$ direction for $x$ or $y$ displacement, and viceversa, but did not apply additional forces in the direction of displacement. To see a clear effect, the magnitude of these three perturbation springs' force constants was chosen as 0.5 of the $z$ component of the regular spring's force constant, and all were oriented so that $T_{x z}$ would be enhanced.

\section{Response to tilting}

The lower (drive) table, as modeled above, is mathematically constrained not to rock or twist, and has a 100 ton mass; the nodes at the base of each of the lowest springs move together with a 100 ton reaction mass. A force applied to the reaction mass results in an acceleration of the base nearly perfectly aligned with the force vector. We measured the response of the real $(78 \mathrm{~kg})$ base table to the drive motors, and found that a nominally horizontal $x$ excitation gave rise to a slight tilting motion (rotation about the $y$ axis) of the base table, causing vertical motion at the table edge of approximately $1 / 10$ the intended horizontal motion. Thus one consequence of our drive method can be simulated by forcing pure tilting of the lower stage and recording the coupling
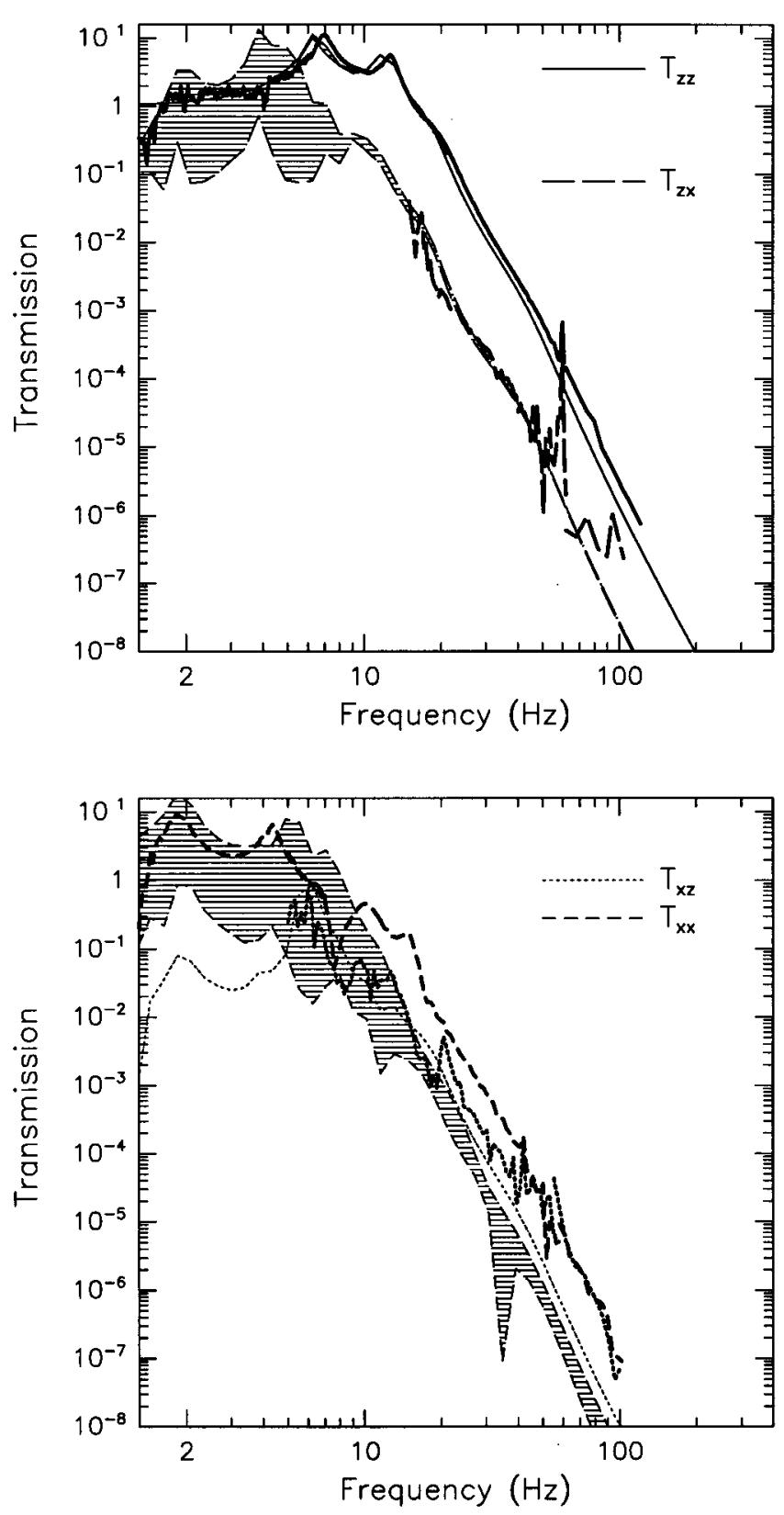

FIG. 4. Measured stack B transmission (bold traces) compared with the ABAQUS model (shaded regions, narrow lines) with the effects of spring and drive vector imperfections added. The shaded ranges allow for the imperfections to add with arbitrary phasing. Note that the allowed range for $T_{x z}$ and $T_{z z}$ is not much more than a line thickness.

of rocking angle, $\theta$, to rocking on the top table, $T_{\theta \theta}$, and the coupling of rocking below to $x$ displacement above, $T_{x \theta}$. From these coefficients one can calculate the contributions to $T_{z x}$ and $T_{x x}$ due to horizontal drive leading to drive table tilts as discussed above. The consequences of the low mass of our drive table were not studied.

\section{Model agreement with data}

In order to check whether a combination of the modeled perturbations can be added together to make a stack model consistent with the measurements, it would be convenient to 
know the exact magnitudes and phases of the perturbations' effects on the real stack. We have estimated the magnitudes to be equal to the values used in the simulations described above. The measurements are not detailed enough to guess at the phases, so the comparison between data and prediction must allow for all possible phases in the addition. Figure 4 compares the addition of the perturbation models with the data. The area shaded represents the range of possible phases between the contributions from the perturbations and the unperturbed (symmetric) model.

\section{Evaluation of FE modeling for this application}

We have attempted to accurately model the effects of a frequency-dependent complex elastic modulus (viscoelasticity) on the spring materials, and the effects of 3-D motion on the measured transmission components.

The success of the viscoelastic modeling can be judged by how well the vertical-vertical transfer function prediction, $T_{z z}$, which is less complicated by other effects, matches the data. We need to compare both the $Q$ 's of the lowfrequency stack modes, which reflect how loss and restoring force compare at the resonance frequencies, and the highfrequency transfer function slope, which reflects the same far above the resonances. The data and models agree fairly well over the entire $T_{z z}$ curve.

The fidelity in three dimensions of the numerical modeling is less satisfactory. In the symmetric stack, the purely vertical normal modes, which number only 8 , are the sole contributors to $T_{z z}$. Thus, a 1-D model is sufficient to understand it. However, the other transfer functions involve the other 52 modes. In a stack with the symmetry broken by random spring strengths or crooked springs, all 60 modes contribute to each of the transfer function components; but for small asymmetries, the non- $T_{z z}$ components will be quite sensitive to the modeling of motions involving all three dimensions. It is evident from Fig. 4 that the predicted ranges of $T_{z z}, T_{z x}$, and $T_{x z}$ are consistent within a few $\mathrm{dB}$ of the data over much of the frequency range. However, although it meets our planned performance goal, the value of $T_{x x}$ is as much as a factor of 10 above the prediction range, exhibiting an extra peak and shoulder between 10 and $20 \mathrm{~Hz}$ before falling off at approximately the same rate as does the range of predictions. We suspect that this might be an artifact of interaction with the drive table, but we cannot be sure without subjecting the system to tests in which all six displacement and angular degrees of freedom, top and bottom, are recorded. Because of the difficulty of this test, we instead plan to install the stack as it will be used and to characterize it in situ, monitoring those degrees of freedom of greatest interest to the application. The additional information from this test will allow us to plan an incomplete, but welltargeted characterization of some "off-diagonal" transfer function terms (e.g., $T_{\theta x}$ ) to improve the agreement of the model with the measurements.

\section{ADDITIONAL WORK}

A stack of this basic design has been incorporated into two LIGO prototype experiments; results are presented in Refs. 20 and 21.

Future work based on this concept will concentrate on optimization of the relative and overall masses to improve the attenuation and to reduce the overall mass. Alternative "lossy spring" elements which meet our vacuum and contamination requirements may also be pursued, with the goal of reducing the effective cutoff frequency and increasing the attenuation fall-off.

\section{ACKNOWLEDGMENTS}

We would like to thank Justin Greenhalgh of Rutherford Appleton Laboratory for discussions and exchanges of stack development results which helped lead us to this design. We would also like to thank the rest of the LIGO team, especially Rainer Weiss and Stanley Whitcomb, for help in carrying out this work. This research was supported by the NSF Grants No. PHY-8803557 and No. PHY-9210038.

${ }^{1}$ A. Abramovici, W. Althouse, R. Drever, Y. Gürsel, S. Kawamura, F. Raab, D. Shoemaker, L. Sievers, R. Spero, K. Thorne, R. Vogt, R. Weiss, S. Whitcomb, and M. Zucker, Science 256, 325 (1992).

${ }^{2}$ C. Bradaschia, R. Del Fabbro, A. Di Virgilio, A. Giazotto, H. Kautzky, V. Montelatici, D. Passuello, A. Brillet, O. Crégut, P. Hello, C. N. Man, P. T. Manh, A. Marraud, D. Shoemaker, J. Y. Vinet, F. Barone, L. Di Fiore, L. Milano, G. Russo, J. M. Aguirregabiria, H. Bel, J. P. Duruisseau, G. Le Denmat, P. Tourrenc, M. Capozzo, M. Longo, M. Lops, I. Pinto, G. Rotoli, T. Damour, S. Bonazzola, J. A. Marck, Y. Gourghoulon, L. E. Holloway, F. Fuligni, V. Iafolla, and G. Natale, Nucl. Instrum. Methods A 289, 518 (1990).

${ }^{3}$ R. Weiss, Q. Prog. Rep. MIT Res. Lab. Electron. 105, 54 (1972).

${ }^{4}$ J. Livas, R. Benford, D. Dewey, A. Jeffries, P. Linsay, P. Saulson, D. Shoemaker, and R. Weiss, in Proceedings of the Fourth Marcel Grossman Meeting on General Relativity, edited by R. Ruffini (North-Holland, Amsterdam, 1986), p. 591.

${ }^{5}$ D. Shoemaker, R. Schilling, L. Schnupp, W. Winkler, K. Maischberger, and A. Rüdiger, Phys. Rev. D 38, 423 (1988).

${ }^{6}$ P. Saulson, Phys. Rev. D 30, 732 (1984).

${ }^{7}$ R. DelFabbro, A. DiVergilio, A. Giazotto, H. Kautzky, V. Montelatici, and D. Passuello, Phys. Lett. A 132, 237 (1988).

${ }^{8}$ M. Stephens, P. Saulson, and J. Kovalik, Rev. Sci. Instrum. 62, 924 (1991).

${ }^{9}$ J. Greenhalgh, internal report of the Rutherford Appleton Laboratory, GEO/207/TCG (1990).

${ }^{10}$ T. Aldcroft, P. Michelson, R. Taber, and R. McLoughlin, Rev. Sci. Instrum. 63, 3815 (1992).

${ }^{11}$ N. Robertson, R. Drever, I. Kerr, and J. Hough, J. Phys. E 15, 1101 (1982).

${ }^{12}$ P. Saulson, Rev. Sci. Instrum. 55, 1145 (1984).

${ }^{13}$ E. J. Douze, Bull. Seismol. Soc. Am. 57, 55 (1967).

${ }^{14}$ P. Saulson, Phys. Rev. D 42, 2437 (1990).

${ }^{15}$ A. Gillespie and F. J. Raab, Phys. Lett. A 190, 213 (1994)

${ }^{16}$ A. Abramovici, T. T. Lyons, and F. J. Raab, Appl. Opt. 34, 183-185 (1995).

${ }^{17}$ L. Ju, D. Blair, H. Peng, and F. van Kann, Meas. Sci. Technol. 3, 463 (1992).

${ }^{18}$ These springs were obtained from the Karman Rubber Company, of Akron, Ohio.

${ }^{19}$ Finite element analysis program ABAQUS, version 4.9 (Hibbit, Karlsson and Sorenson, Inc., Providence, RI).

${ }^{20}$ L. A. Sievers and R. E. Spero, in Proceedings of the Seventh Marcel Grossmann Meeting, edited by R. Ruffini and M. Keiser (World Scientific, Singapore, to be published)

${ }^{21}$ A. Abramovici, W. Althouse, J. Camp, D. Durance, J. Giaime, A. Gillespie, S. Kawamura, A. Kuhnert, T. Lyons, F. Raab, R. Savage, D. Shoemaker, L. Sievers, R. Spero, R. Weiss, S. Whitcomb, and M. Zucker, submitted to Science. 